\title{
Evaluation du comportement de variétés améliorées de manioc riches en bêta-carotène au Sud du Bénin
}

\author{
Adijatu Kouboura Alice DJINADOU*, Nestor Isséré OLODO et \\ Adolphe ADJANOHOUN
}

Centre de Recherches Agricoles Sud, Institut National des Recherches Agricoles du Bénin (INRAB)

*Auteur correspondant ; E-mail : djinadoualice@ gmail.com, Tél. (+229) 95062963 ; 96414024

\section{RESUME}

Le manioc, tubercule le plus consommé du Bénin, peut contribuer à résorber les problèmes de carence en vitamine A. L'étude visait à évaluer le rendement et la teneur en bêta-carotène de 15 variétés de manioc fortifiées en bêta-carotène comparées à la variété RB89509 dans les conditions agroécologiques du Sud-Bénin et à évaluer la résistance de ces variétés aux attaques parasitaires. Le dispositif expérimental est constitué d'un bloc aléatoire complet avec 16 variétés et 4 répétitions. L'analyse de la variance a été exécutée, le rendement en racines calculé puis la teneur en bêta-carotène estimée par variété. Les résultats ont montré que les cinq variétés adaptées aux conditions agroécologiques et phytosanitaires du Sud-Bénin sont I011412, I070539, I090090, I083594 et I090151 avec des taux en bêta-carotène et des rendements en racines respectifs de $(9,82$ $\mu \mathrm{g} / \mathrm{g} ; 23,68 \mathrm{t} / \mathrm{ha}),(9,7 \mu \mathrm{g} / \mathrm{g} ; 34,34 \mathrm{t} / \mathrm{ha}),(9,69 \mu \mathrm{g} / \mathrm{g} ; 23,45 \mathrm{t} / \mathrm{ha}),(9,23 \mu \mathrm{g} / \mathrm{g} ; 30,42 \mathrm{t} / \mathrm{ha})$ et $(9,16 \mu \mathrm{g} / \mathrm{g}$; 33,29 t/ha). La variété locale, RB89509, a présenté le taux en bêta-carotène le plus faible, de 2,97 $\mu \mathrm{g} / \mathrm{g}$ avec un faible rendement de 15,28 t/ha. Ces cinq variétés méritent une vulgarisation en milieu paysan selon une approche participative par les décideurs.

(C) 2018 International Formulae Group. All rights reserved.

Mots clés: Manihot esculenta, Vitamine A, sécurité alimentaire, biofortification, Bénin.

\section{Assessment of the behavior of cassava improved varieties rich in beta- carotene in the South of Benin}

\begin{abstract}
Cassava, the most widely consumed tuber in Benin, can help reduce vitamin A deficiency. The study aimed at evaluating the yield and beta-carotene content of 15 cassava fortified cassava varieties compared with the variety RB89509 in the agroecological conditions of southern Benin and at evaluating the resistance of these varieties to parasitic attacks. The experimental set up consists of a complete random block with 16 varieties and 4 repetitions. The analysis of the variance was performed, the roots yield was calculated then the beta-carotene content was estimated for each variety. The results showed that the five varieties adapted to the agroecological and phytosanitary conditions of southern Benin were I011412, I070539, I090090, I083594 and I090151 with beta-carotene levels and respective root yields of $(9.82 \mu \mathrm{g} / \mathrm{g} ; 23.68 \mathrm{t} / \mathrm{ha}),(9.7 \mu \mathrm{g} / \mathrm{g}, 34.34 \mathrm{t} / \mathrm{ha})$, (9.69 $\mu \mathrm{g} / \mathrm{g}, 23.45 \mathrm{t} / \mathrm{ha}),(9.23 \mu \mathrm{g} / \mathrm{g} ; 42 \mathrm{t} / \mathrm{ha})$ and $(9.16 \mu \mathrm{g} / \mathrm{g}, 33.29 \mathrm{t} / \mathrm{ha})$. The local variety, RB89509, had the lowest beta carotene level of $2.97 \mu \mathrm{g} / \mathrm{g}$ with a low yield of $15.28 \mathrm{t} / \mathrm{ha}$. These five best varieties deserve to be popularized in rural areas by decision-makers, according to a participative approach.
\end{abstract}

(c) 2018 International Formulae Group. All rights reserved.

Keywords: Manihot esculenta, vitamin A, food security, biofortification, Benin. 


\section{INTRODUCTION}

La production mondiale de manioc, Manihot esculenta, a augmenté de 4,6\% entre 2013 et 2014 avec le Nigeria comme le plus grand producteur de l'Afrique (El-Sharkawy, 2003). Ses racines, accessibles à moindre coût, sont très riches en amidon. Il est la deuxième source d'énergie alimentaire dans les pays en développement (Silva et al., 2008 ; Salvador et al., 2014) où les problèmes de malnutrition sont récurrents. Il est consommé sous plusieurs formes : farine, granulés, fufu, etc. Les feuilles sont consommées comme sauce légume en Afrique (Tanumihardjo et al., 2010) et particulièrement au Bénin où elles servent aussi d'emballage (Dansi et al., 2008 ; Amoussa-Hounkpatin et al., 2012). Ainsi, le manioc joue un rôle prépondérant dans la sécurité alimentaire des populations les plus pauvres (Djoulde, 2005), servant de nourriture principale à plus de 600 millions de personnes (Wydra et Verdier, 2002).

Toutefois, du fait que les racines et les tubercules sont très pauvres en lipides, ce ne sont pas de bonnes sources de vitamines liposolubles. En effet, les racines et les tubercules contiennent généralement très peu de bêta-carotène à l'exception de certaines variétés de patate et de couleur foncée dont une dose de $13 \mathrm{~g}$ par jour suffirait à couvrir le besoin de vitamine A (Gannon et al., 2016; Toulsoumdé Songre-Ouattara et al., 2016). De même, il existe des variétés d'igname très colorées, notamment D. cayenensis, appelées igname jaune. Cette couleur jaune est due aussi au bêta-carotène dans des quantités allant de 0,14 à $1,4 \mathrm{mg} / 100 \mathrm{~g}$ (Gannon et al., 2016).

Le bêta-carotène, représentant le plus connu de la provitamine A et un des plus répandus dans la nature et dans notre alimentation, est d'une grande famille de nutriments appelés caroténoïdes. Des études réalisées en Amérique du Nord ont permis d'établir une relation d'équivalence entre le taux de bêta-carotène et le taux de vitamine $\mathrm{A}$ dans le sang chez les êtres humains. Le bêtacarotène est essentiel pour l'accomplissement de multiples fonctions chez l'homme, telles que la vision, la croissance, la reproduction, l'immunité (Tanumihardjo, 2011 ; La Frano et al., 2013; Tanumihardjo, 2013). Au Nigéria, par exemple, où le manioc est le principal aliment de base, la prévalence globale de la carence en vitamine A touche environ $30 \%$ des enfants âgés de 0 à 59 mois. La carence en vitamine A affecte environ 127 millions d'enfants d'âge préscolaire et 7 millions de femmes enceintes avec l'Afrique en pole position ou la quasi-totalité des pays a une prévalence très élevée et marquée par une carence sévère en vitamine A (Michaud et al., 2000). Selon le rapport sur le profil nutritionnel du Bénin (FAO, 2011), les données disponibles à ce jour sur la carence en vitamine A des jeunes enfants indiquent une déficience plus marquée dans le Nord $(82,8 \%)$ que dans le Sud $(63,6 \%)$ du pays puis dans les milieux ruraux que dans les milieux urbains (Amoussa-Hounkpatin et al., 2012).

En Afrique, des efforts de recherche et de diffusion de technologies ont été réalisés, entre autres, sur la maîtrise des pratiques culturales (Kouadio et al., 2011 ; Faye, 2015), la lutte contre les maladies et les parasites (Lokko et al., 2012), mais aussi et surtout l'utilisation de bonnes variétés afin d'améliorer le potentiel et la diversité de la spéculation. Mokemiabeka et al. (2011) ont montré que la variabilité des caractéristiques des produits obtenus à partir de racines de manioc roui est le reflet de plusieurs facteurs, dont la variété de manioc, les conditions environnementales et la méthode de rouissage.

$\mathrm{Au}$ Bénin, plusieurs travaux de recherches sont réalisés et s'inscrivent dans le cadre des systèmes de production (Adjanohoun, 2006), Adjanohoun et Allagbé (2011), la lutte phytosanitaire (Fagbemissi et al., 2002), l'utilisation de variétés améliorées et de vitroplants afin d'améliorer le potentiel et la diversité de la spéculation (Ahanhanzo et al., 2009 ; Cacaï et al., 2012 ; Leyva-Guerrero et al., 2012).

Le Bénin compte 116 accessions de manioc (Agre et al., 2015). Les variétés les plus vulgarisées sont des variétés à chair blanche et, par conséquent, pauvres en 
vitamine A (Gannon et al., 2016). Parmi ces variétés, BEN 86052, RB 89509 et TMS 30572 sont adoptées par les producteurs depuis plusieurs décennies. D'un potentiel de production de $45 \mathrm{t} / \mathrm{ha}$, leurs rendements au niveau paysan varient entre 7 et 10 t/ha (Adjanohoun et Allagbé, 2011). Or, au vu de son importance dans l'alimentation de nombreux pays subsahariens et principalement au Bénin, le manioc pourrait servir de vecteur très efficace pour disséminer la provitamine A aux populations souffrant ou risquant de souffrir de carences en cette vitamine, surtout les enfants (Sahouegnon et al., 2014). Il est alors important de trouver des variétés de manioc riches en vitamine $\mathrm{A}$ et à haut rendement. L'objectif scientifique de cette étude est d'évaluer le rendement et la teneur en bêta-carotène de quinze (15) variétés fortifiées en bêta-carotène dans les conditions agroécologiques du Sud-Bénin et d'évaluer la résistance de ces quinze (15) variétés face aux attaques des parasites du manioc. L'objectif de développement est d'améliorer la sécurité alimentaire et nutritionnelle des populations au Bénin et dans la sous-région.

\section{MATERIEL ET METHODES}

\section{Zone d'étude}

L'étude a été réalisée dans la région méridionale au Sud du Bénin caractérisée par un climat subéquatorial avec une forte humidité et une alternance de deux saisons sèches et de deux saisons pluvieuses. La pluviométrie moyenne est de $1200 \mathrm{~mm}$ répartis sur 8 mois (mai à novembre). Les précipitations maximales sont enregistrées au cours des mois de juin et octobre et les minimales au cours du mois d'août. La température moyenne est autour de $27{ }^{\circ} \mathrm{C}$ (Adjanohoun et Allagbé, 2011). Le sol est de type ferralitique rougeâtre, sans concrétions (Aïhou, 2003). Sa teneur moyenne en matière organique et en phosphore assimilable est respectivement de $1.03 \%$ et $9 \mathrm{ppm}$. Les teneurs en bases échangeables sont de 0,14 méq/100 g de sol pour le potassium, 2,59 méq/100 g pour le calcium, 0,59 méq/100 g pour le magnésium et $0,23 \mathrm{méq} / 100 \mathrm{~g}$ pour le sodium (Agbodjato et al., 2015).

\section{Matériel végétal}

Le matériel biologique de plantation était constitué de 16 variétés de manioc, dont 15 variétés biofortifiées par l'Institut International d'Agriculture Tropicale (IITA) au Nigeria et une variété locale améliorée. Les 15 variétés produites par l'IITA-Ibadan et utilisées dans le cadre de cette étude étaient les suivantes : I011412 ; I070557 ; I082461 ; I090090 ; I090151 ; I083703 ; I083594 ; I085392; I083774； I070593； I011797; I083724 ; I070539 ; I061635 ; I082264. Leur cycle végétatif est de 12 mois, avec des rendements potentiels variant entre 35 et 45 t/ha et des teneurs potentielles en bêtacarotène allant de 12 à $18 \mu \mathrm{g} / \mathrm{g}$. La dernière variété considérée comme témoin était la variété locale améliorée RB89509, largement vulgarisée au Bénin et adoptée par les producteurs, les transformateurs et les consommateurs depuis près de deux décennies. Le cycle végétatif de cette variété de manioc est de 16 à 18 mois avec des rendements potentiels de 25 à 30 t/ha (Adjanohoun, 2006).

\section{Dispositif expérimental}

Les variétés de manioc ont été réparties au hasard dans quatre (4) répétitions selon le dispositif de FISHER ou dispositif des blocs complets randomisés sur une superficie de $1920 \mathrm{~m}^{2}$ (64 m x $\left.30 \mathrm{~m}\right)$ pendant deux campagnes consécutives (2014 - 2015 et 2015-2016). Les dimensions de la parcelle élémentaire étaient de $22,4 \mathrm{~m}^{2}(5,6 \mathrm{~m} \times 4 \mathrm{~m})$. Une allée de $2 \mathrm{~m}$ a été aménagée entre deux répétitions et une autre de $1 \mathrm{~m}$ entre deux parcelles élémentaires. Chaque variété a occupé 4 lignes de 5,6 $\mathrm{m}$ de long dont 2 lignes de bordure et 2 lignes centrales ou utiles. La distance entre les lignes était de $1 \mathrm{~m}$ et celle sur la ligne était de $0,8 \mathrm{~m}$, ce qui donne une densité de plantation de 28 boutures par parcelle élémentaire pour une densité totale de 12.500 boutures par hectare. Les essais ont été effectués sur deux campagnes culturales sans apport d'intrants. 
La plantation à plat a été faite manuellement durant la grande saison des pluies au mois de juillet 2014 pour la première année, puis à la même période en 2015 pour la seconde année. Des boutures saines ont été plantées à l'oblique et recouvertes de sol jusqu'au $2 / 3$ de leur longueur de manière à avoir des nœuds sur la partie aérienne. Au total, 1792 boutures saines de 20 à $25 \mathrm{~cm}$ ont été réparties sur les quatre répétitions de la parcelle expérimentale, soit 112 boutures par variété. Aucun engrais ni insecticide n'a été utilisé pendant tout le cycle cultural.

\section{Figure 1: Dispositif expérimental}

\section{Collecte de données}

Les données collectées sont d'ordre agronomique, phytosanitaire et post-récolte. Il était question des données qualitatives et quantitatives. Des observations étaient faites sur les plants de manioc tous les 15 jours. L'attention a été mise sur le développement végétatif des plants et sur l'apparition des maladies. Les symptômes observés ont été notés. Les données agronomiques considérées avant la récolte sont le nombre de plants à la levée, le nombre de poussées sur toute la surface emblavée par génotype, l'observation de la mosaïque du manioc et de la ruine bactérienne du manioc à 1 mois, 3 mois et 6 mois, puis de l'anthracnose et de l'acarien vert à 6 mois et 9 mois. Les scores utilisés pour qualifier les symptômes des maladies sont les suivants : 1 = zéro attaque (absence de symptômes), 2 = faible attaque (rares traces de la maladie), $3=$ attaque moyenne (quelques traces de la maladie), $4=$ attaque élevée (plusieurs feuilles présentant des symptômes) et $5=$ attaque très élevée (rabougrissement des plants et arrêt de la croissance). La récolte a été faite 12 mois après plantation. Les données post-récolte ont été collectées par variété et par parcelle à savoir, le nombre de plants récoltés, le nombre de racines, le poids en kilogramme des racines fraîches, le poids de la partie aérienne, la taille des racines classée en petite, moyenne et grande à la récolte avec l'échelle suivante : $3=$ petite; $5=$ moyenne et $7=$ grande. La forme des racines a été examinée visuellement et marquée à la récolte en utilisant les paramètres suivants : $1=$ Conique; $2=$ cylindro-conique; $3=$ cylindrique; 4 = fusiforme; $5=$ irrégulière et 6 = Combinaison de plusieurs formes. Pour le caractère longueur du cou, le pédoncule des racines a été marqué en utilisant les codes suivants : $0=$ absence; $3=$ court; $5=$ intermédiaire et $7=$ longue ;

Le nombre de racines pourries a été compté dans chaque parcelle et par variété. La couleur de la pulpe (cortex) de la racine a été examinée immédiatement après son ouverture a été examinée visuellement et enregistrée comme suit: 1 = blanc ou crème; $2=$ jaune . La couleur de la peau intérieure des racines a été examinée visuellement et enregistrée comme suit : 1 = blanc ou crème; 2 = jaune; 3 = rose et $4=$ violet. La facilité d'épluchage a été déterminée par trois personnes différentes et leur conclusion a été enregistrée pour chaque génotype. L'échelle suivante a été utilisée: 3 = Facile à peler; 2 = modérément facile à enlever et $1=$ Difficile à enlever.

Le taux en bêta-carotène a été déterminé au laboratoire avec un appareil électronique appelé iCheck-Carotene. La méthode utilisée est celle mise au point par l'IITA - iCheck ${ }^{\mathrm{TM}}$ Carotène - introduite par BioAnalyt, qui est utilisée pour cribler rapidement de grandes populations (Hernan et Parkes, 2015). Le kit de test est constitué d'un photomètre portable et de flacons de réactifs prêts à l'emploi. Cette combinaison permet un filtrage rentable, simple, convivial et rapide de grands nombres d'échantillons, y compris sur les lieux de travail sans électricité ni réfrigération. Les racines récoltées le matin ont été étiquetées, puis lavées et épluchées. Des échantillons ont été préparés et $0,4 \mathrm{ml}$ a été prélevé de l'échantillon de suspension et injecté dans le flacon de réactif (iEx Carotene) inclus dans le kit de test. Le flacon a été secoué et laissé au repos pendant au moins cinq minutes pour l'extraction des caroténoïdes avant que la mesure ne soit prise. La lecture des données a été effectuée 60 minutes après, ce qui a permis aux caroténoïdes de se stabiliser dans le flacon. Le flacon a été inséré dans l'appareil et mesuré. 
L'appareil a affiché le résultat en $\mathrm{mg}$ de caroténoïdes par litre $(\mathrm{mg} / \mathrm{l})$. Pour obtenir la concentration totale en caroténoïdes (TCC) dans la racine de manioc, le résultat affiché a été multiplié par le facteur de dilution (volume total de l'échantillon dans l'eau). A chaque mesure de l'appareil, l'inscription à l'écran correspondant au taux de bêtacarotène est enregistrée sur une fiche de collecte préétablie.

\section{Analyse des données}

Le rendement en racines fraîches de manioc a été déterminé par la formule suivante :

Rdmt $(\mathrm{t} / \mathrm{ha})=\left(P M_{l} R T\right) x(N M R T) x$ $(N P / h a) \times 10^{-6}$ où $P M_{1} R T=$ poids moyen $(\mathrm{g})$ d'une racine; $N M R T=$ nombre moyen de racines par plant $; N P / h a=$ nombre de plants par hectare $; 10^{-6}=$ facteur de conversion du rendement en t/ha car le $P M_{l} R T$ est exprimé en grammes.

\section{Analyses statistiques}

Pour la caractérisation des variétés, l'analyse de variance a été faite avec la
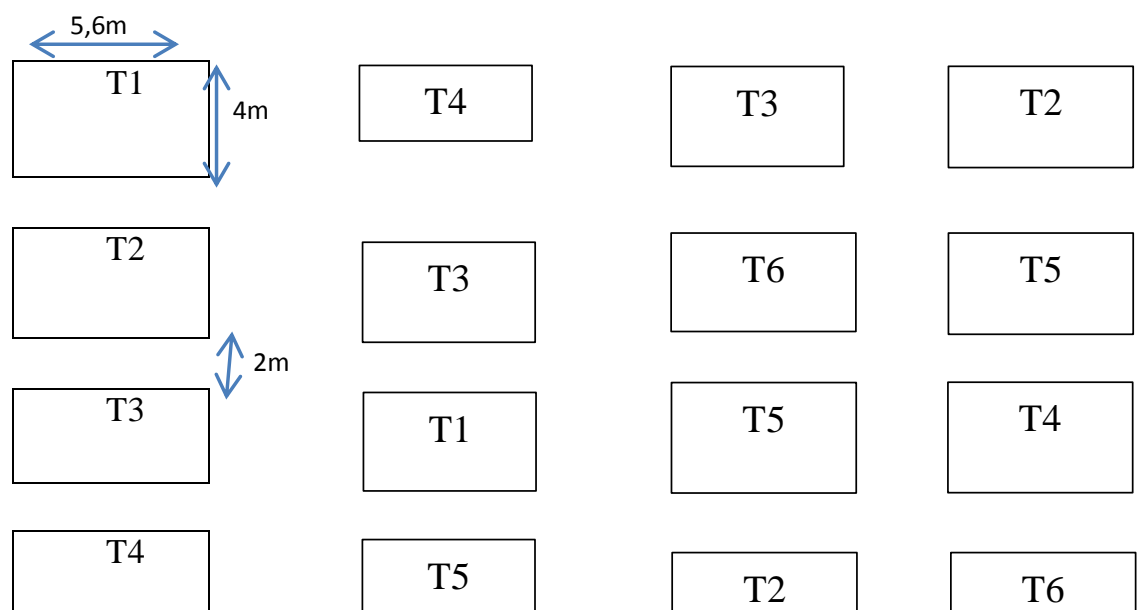
Canonique Discriminante pas à pas (CDA) a été réalisée pour identifier les paramètres de sélection les plus pertinents qui caractérisent les variétés de manioc étudiées (NRt, RtWt, ShtWt, WtAir, WtH20 et Betac) et ceci par année (2014 et 2015).

L'effet des variétés et du temps a été testé sur le logarithme de la teneur en bêtacarotène, retenue comme la seule variable pertinente commune aux deux facteurs. Ainsi, l'Analyse de la Variance (ANOVA) à deux facteurs fixes croisés a été réalisée. Un diagramme d'interaction a été réalisé pour évaluer la différence de combinaison entre les deux facteurs. Le seuil de significativité est de 0,05 .

L'analyse canonique discriminante pas à pas a été réalisée avec la fonction 'greedy.wilks" du package ('KlaR'). L'analyse canonique discriminante a été effectuée à l'aide de la fonction "candisc" du package ('candisc') et le test SNK, grâce à la fonction 'HSD.test', packages ('agricolae') du logiciel R 3.3.2 (R Development Core Team. 2016).
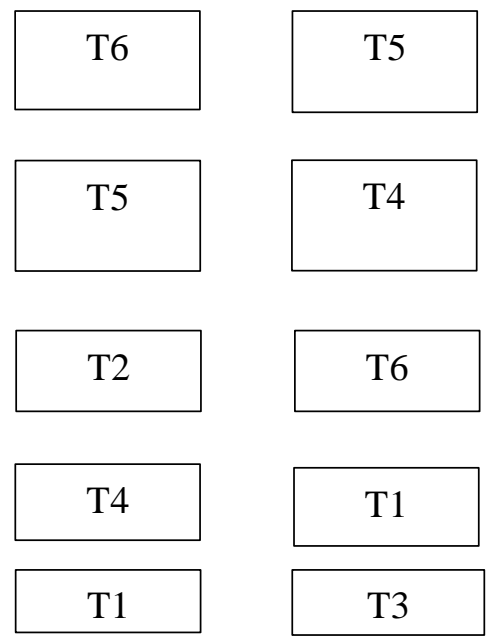

Figure 2: Dispositif expérimental. 


\section{RESULTATS}

Rendement et teneur en bêta-carotène des 15 variétés de l'IITA et de la variété locale améliorée

L'estimation du rendement en racines fraîches à l'hectare et du taux de bêtacarotène dans les racines (Tableau 1) montre une variation de ces facteurs au sein des variétés biofortifiées en bêta-carotène et entre ces variétés et la variété locale RB89509. Onze (11) des quinze (15) variétés biofortifiées en bêta-carotène de l'IITAIbadan ont un rendement en racines fraîches à l'hectare relativement supérieur à celui de la variété locale améliorée RB89509 qui a donné un rendement de 15,28 t/ha dans les mêmes conditions de culture. Parmi ces 11 variétés, la variété I082461 a le rendement le plus élevé, estimé à 36,74 t/ha et la variété I085392 qui a un rendement proche de celui de la variété locale RB89509. La teneur en bêta-carotène de toutes les 15 variétés biofortifiées est supérieure à celle de la variété RB89509. Les variétés I011412, I070539, I090090, I083594 et I090151 sont les cinq variétés à fort taux de bêta-carotène (en moyenne $9 \mu \mathrm{g} / \mathrm{g}$ ) avec un rendement en racines fraîches à l'hectare nettement supérieur à celui de la variété RB89509. L'analyse statistique de ces caractères quantitatifs et des caractères qualitatifs étudiés montre une différence significative $(\mathrm{P}<0,05) \quad$ des racines entre variétés biofortifiées en bêta-carotène de manioc en termes de rendement à l'hectare, de la couleur interne de la pulpe, de la couleur de la peau, de la facilité d'épluchage et du pilonnement. Ces caractères se sont tous révélés plus intéressants au niveau des quinze variétés biofortifiées importées de l'IITA-Ibadan que chez la variété locale améliorée RB89509 sauf le caractère épluchage qui s'est révélée difficile pour toutes les variétés sans exception. Les caractères tels que la forme des racines, la longueur du pédoncule, le poids de la partie aérienne, la pourriture des tubercules ne varient pas significativement entre les variétés de manioc. La forme cylindro-conique est la forme dominante des racines et les pédoncules sont courts d'une variété à une autre. La variété I070593 est celle qui a donné des racines de plus petite taille, suivie de la variété I070557 puis de la variété locale RB89509. La variété I070539, quant à elle, a produit les plus grandes racines, suivie des variétés I082461, I083724, I061635 et I090151.

Concernant le pilonnement, presque toutes les variétés de manioc ont un niveau de farinage faible qui varie entre 1 et 1,5 sauf les variétés I061635 et I082264 qui ont un niveau de farinage plus élevé, à savoir 3 et 2,3 respectivement.

L'Analyse Canonique Discriminante des six variables a montré que seules les variables NRt (Nombre de racines), RtWt (Poids des racines) et le Betac (bêta-carotène) sont significativement $(\mathrm{P}<0,05)$ discriminantes (Tableau 2) et donc les plus pertinents pour discriminer les 16 variétés en 2014 et en 2015.

L'effet des variétés et du temps testé sur le logarithme de la teneur en bêta-carotène par l'analyse de la variance (ANOVA) à deux facteurs fixes croisés (Tableau 3) montre une différence significative $(\mathrm{P}<0,05)$ entre les variétés en ce qui concerne la teneur en bêtacarotène. Cette différence est très hautement significative $(\mathrm{P}<0,05)$ lorsque le facteur temps est croisé à la variété.

Le diagramme d'interaction entre variétés et le temps suivant la valeur en bêtacarotène (Figure 2) révèle une forte différenciation entre les variétés en 2015 qu'en 2014. La variété I011412 est la meilleure suivie des variétés I070539, I090090, I083594 et I090151 qui ont à la fois des rendements et des poids moyens en racines intéressants. Par contre, quant à la variété locale, elle a la plus faible valeur logarithmique moyenne de la teneur en bêtacarotène.

\section{Résistance des variétés biofortifiées et de la variété locale aux parasites du manioc}

Dans l'ensemble, toutes les variétés améliorées tout comme la variété locale sont quasiment attaquées dans les mêmes proportions par les diverses maladies à savoir, la mosaïque du manioc (CMD), la ruine 
bactérienne du manioc (CBB), l'acarien vert et l'anthracnose (CAD) (Figures 4, 5, 6 et 7). Les différentes attaques n'ont pas atteint un niveau sévère puisqu'elles sont soit faibles ou moyennes. Toutes les variétés ont été très résistantes face à la mosaïque du manioc le premier mois après la mise en place des plantations, mais au troisième mois elles ont été toutes faiblement attaquées (Figure 3). Dans le cas de l'acarien vert, l'attaque a été élevée au deuxième mois mais très faible au premier et troisième mois (Figure 4). L'attaque des différentes variétés par la ruine bactérienne s'est fait sentir au troisième et sixième mois mais le niveau d'attaque reste faible (Figure 5). Aussi, l'attaque des variétés par l'anthracnose est faible à partir du sixième mois (Figure 6). Toutefois, il est à remarquer que les variétés I090090 et I011412 sont un peu plus sensibles aux différentes maladies comparativement aux autres variétés.

Tableau 1 : Variabilité des caractères quantitatifs des variétés biofortifiées en bêta-carotène et de la variété locale.

\begin{tabular}{lcc}
\hline Variétés & Rendement moyen (t/ha) & $\begin{array}{c}\text { Taux en bêta- } \\
\text { carotène }(\boldsymbol{\mu g} / \mathbf{g})\end{array}$ \\
\hline I082461 & 36,74 & 7,14 \\
I070539 & 34,34 & 9,7 \\
I011797 & 33,37 & 4,32 \\
I090151 & 33,29 & 9,16 \\
I083594 & 30,42 & 9,23 \\
I061635 & 27,33 & 7,94 \\
I082264 & 27,19 & 7,48 \\
I083774 & 26,91 & 6,3 \\
I011412 & 23,68 & 9,82 \\
I090090 & 23,45 & 9,69 \\
I083724 & 19,26 & 7,56 \\
RB89509 & 15,27 & 2,97 \\
I070557 & 14,14 & 7,76 \\
I085392 & 12,71 & 8,85 \\
I083703 & 11,48 & 8,74 \\
I070593 & 4,77 & 11,03 \\
\hline
\end{tabular}

Tableau 2: Analyse Canonique Discriminante des six variables réponses (NRt, RtWt, ShtWt, WtAir, $\mathrm{WtH}_{2} \mathrm{O}$ et $\mathrm{Betac}$ ) suivant les variétés.

\begin{tabular}{lccccr}
\hline Variables & Wilks. lambd: & $\begin{array}{c}\text { F. statistics. } \\
\text { Overall }\end{array}$ & p. value. overal F. & statistics. dil p. value. dif \\
\hline sqrt(NRt) & 0,18362 & 4,742 & 0,00181737 & 4,742 & 0,001817 \\
$\log ($ RtWt $)$ & 0,03617 & 4,258 & 0,00007783 & 4,077 & 0,004086 \\
$\log ($ Betac $)$ & 0,2684 & 2,907 & 0,02082 & 2,907 & 0,02082 \\
\hline
\end{tabular}


Tableau 3: Comparaison du logarithme du taux moyen de bêtacarotène suivant les variétés étudiées et le temps d'étude.

\begin{tabular}{lccccc}
\hline Source & Df & SumSq & MeanSq & F value & $\operatorname{Pr}(>\mathbf{F})$ \\
\hline Variété & 15 & 3.39 & 0.226 & 2.31 & $0.0231 *$ \\
Temps & 1 & 0.02 & 0.022 & 0.23 & $0.6373 \mathrm{~ns}$ \\
Variété Temps & 15 & 5.03 & 0.335 & 3.42 & $0.0017 * *$ \\
Residuals & 32 & 3.14 & 0.098 & & \\
\hline ** indiquent une signification à 1\%, & $*$ indiquent une signification à $5 \%$, & ns indique une non signification
\end{tabular}

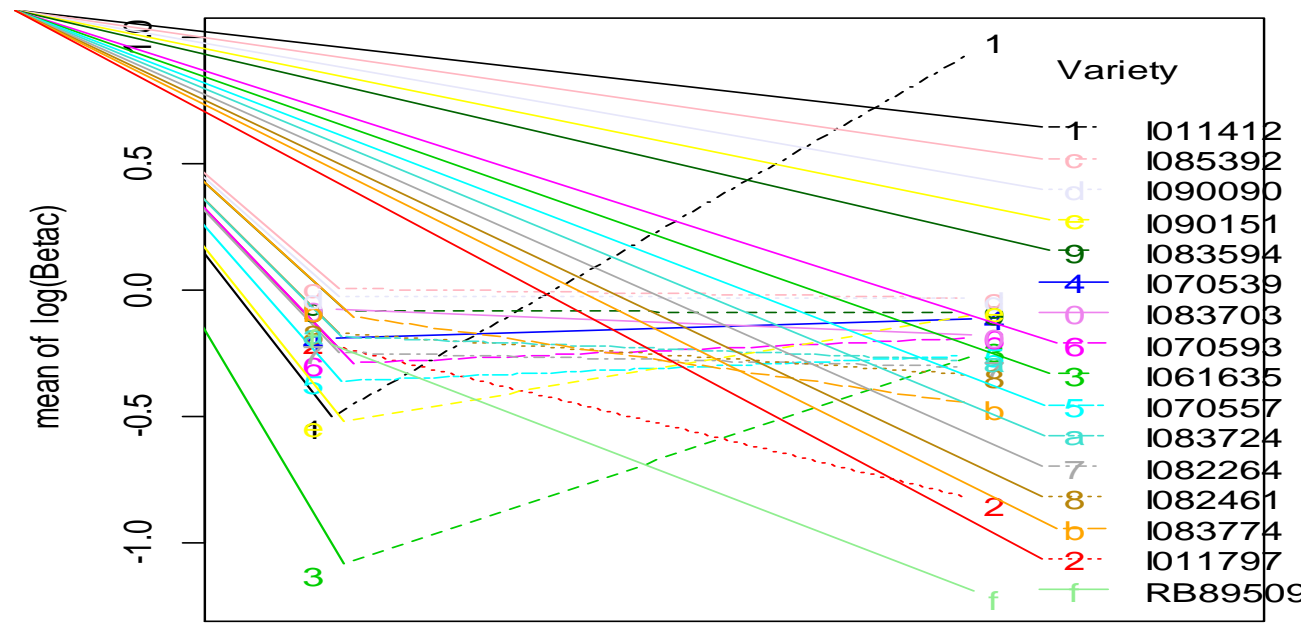

T1

T2

Temps

Figure 2. Diagramme d'interaction entre les variétés et le temps suivant les valeurs logarithmiques moyennes de la teneur en bêta-carotène.

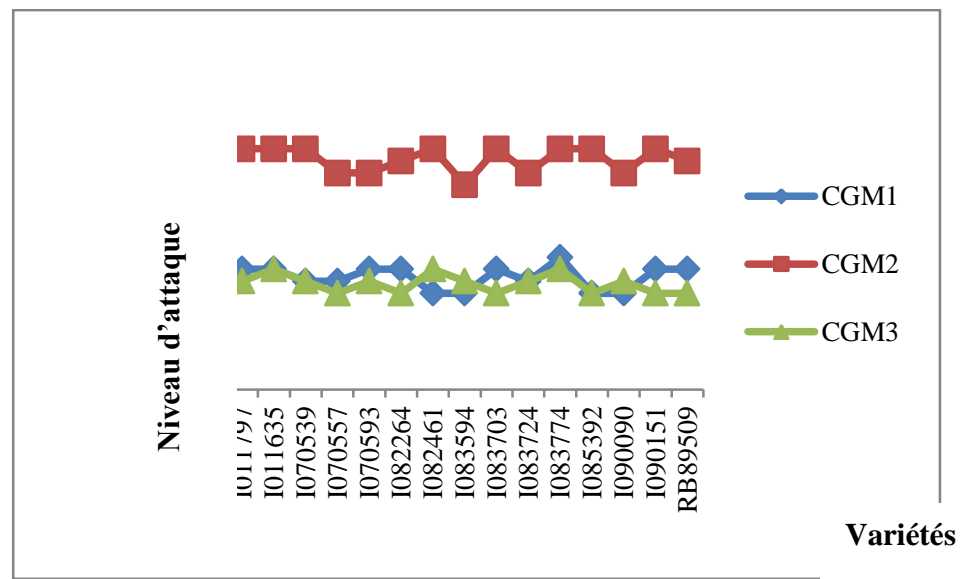

Figure 3: Niveau d'attaque des 16 variétés de manioc par l'acarien vert (CGM).

CGM1 : données sur les symptômes à 1 MAP (Mois Après Plantation); CGM2 : données sur les symptômes à 2 MAP; CGM3 : données sur les symptômes à 3 MAP. 


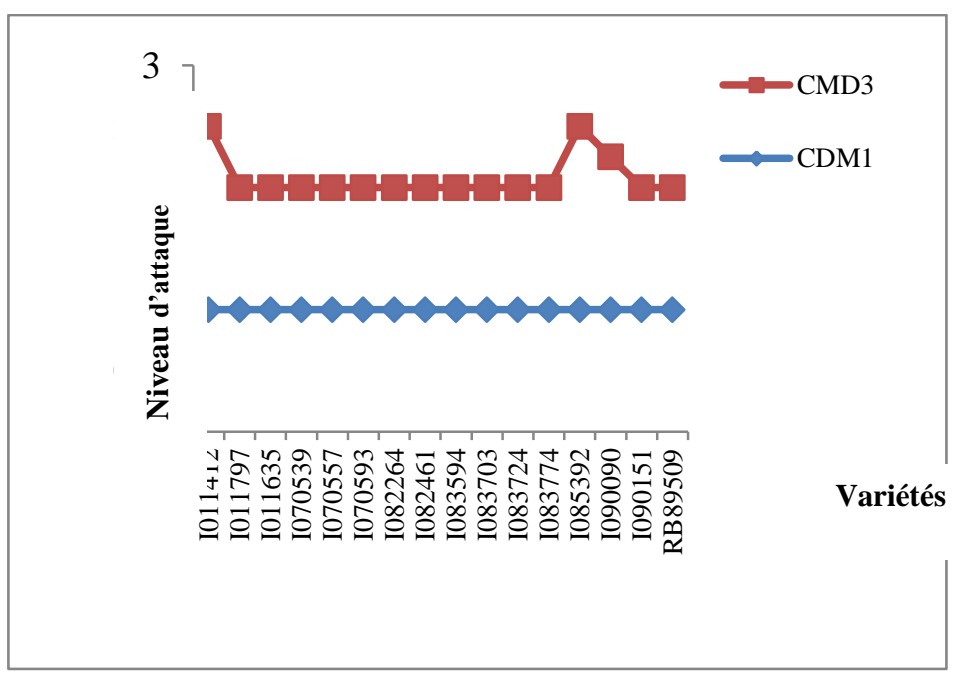

Figure 4 : Niveau d'attaque des 16 variétés par la mosaïque du manioc (CMD). CMD1 : données sur les symptômes à $1 \mathrm{MAP} ; \mathrm{CMD} 3$ : données sur les symptômes à 3 MAP.

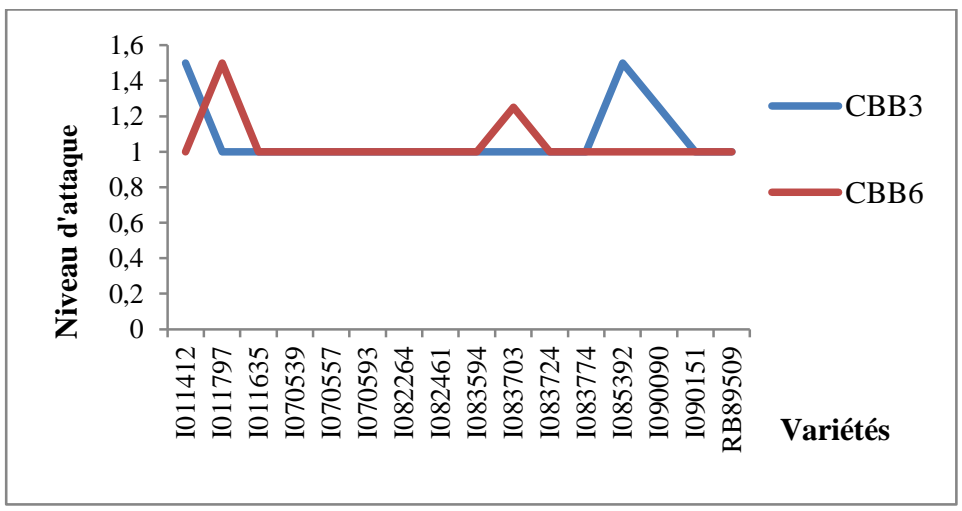

Figure 5: Niveau d'attaque des 16 variétés par la ruine bactérienne (CBB). $\mathrm{CBB} 3$ : données sur les symptômes à $3 \mathrm{MAP}$; $\mathrm{CBB} 6$ : données sur les symptômes à 6 MAP .

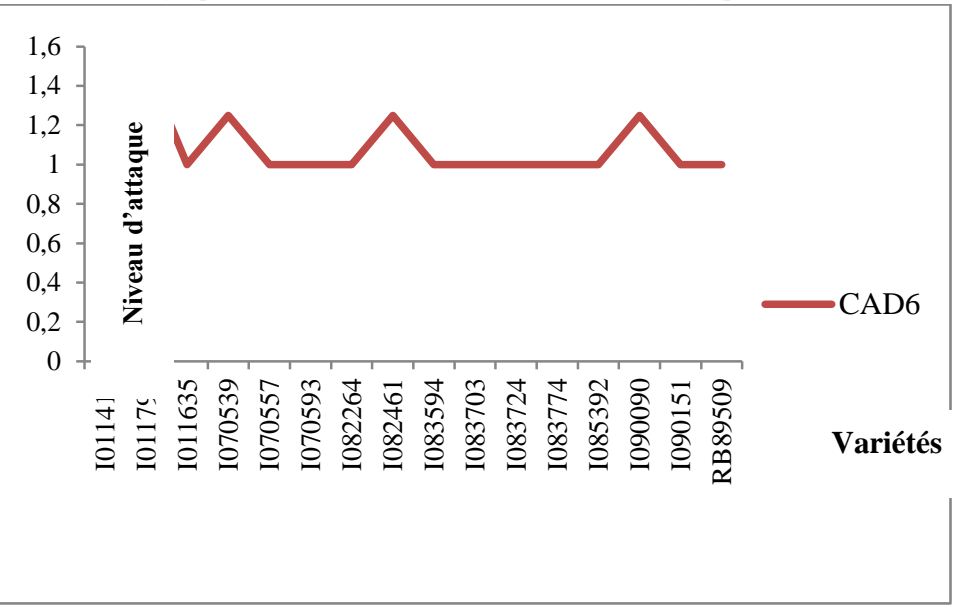

Figure 6 : Niveau d'attaque des 16 variétés par l'anthracnose (CAD) CAD6 : données sur les symptômes à 6 MAP. 


\section{DISCUSSION}

Un total de 11 variétés de manioc biofortifiées en bêta-carotène a donné un rendement en racines à l'hectare généralement élevé par rapport à la variété RB89509 vulgarisée et adoptée fortement par les producteurs de manioc dans le pays. Ces résultats sont en accord avec les travaux de Wydra et Verdier (2002), N'Zué et al. (2004), Bakayoko et al. (2012) et Kouadio et al. (2014). Ces auteurs unanimement s'accordent sur le fait que les variétés améliorées donnent des rendements significativement supérieurs à ceux des variétés locales. La majorité de ces variétés a montré une adaptation aux conditions agroécologiques de la région sud du Bénin. Ces résultats confirment la distribution du manioc dans la zone tropicale qui constitue une zone favorable au développement des cultures du manioc. Dans l'ensemble, les variétés biofortifiées en bêtacarotène ont un taux en bêta-carotène élevé, entre 4,32 et $11,03 \mu \mathrm{g} / \mathrm{g}$, contrairement aux traces de vitamine ou de bêta-carotène généralement retrouvé dans les variétés non améliorées et plus spécifiquement dans la variété améliorée RB89509 du Bénin (2,97 $\mu \mathrm{g} / \mathrm{g})$. Ces résultats confortent les observations faites par Gannon et al. (2016) sur la variation de la couleur de la pulpe des racines suivant la teneur en bêta-carotène. Toutes les variétés biofortifiées en bêtacarotène ont une pulpe de couleur jaune contrairement à la variété RB89509 dont la pulpe était blanche. Mais il est important de remarquer que le potentiel escompté pour chacune de ces variétés, en termes de rendement et de la teneur en bêta-carotène, n'a pas été atteint.

Si l'analyse canonique discriminante a différencié la variété I011412 des autres, c'est parce qu'elle est la variété la plus riche en bêta-carotène et disposant d'un rendement à l'hectare de même que d'un poids moyen en racines fraîches très intéressants. La valeur de la pourriture des tubercules a été faible voire nulle. Les autres caractères comme la résistance à la récolte, le diamètre, la longueur, le nombre de tubercules par plant et le poids frais individuel des racines dépendent des variétés et des conditions écologiques (Kouadio et al., 2014). Au niveau des caractères morphologiques des racines, les caractères «longueur du cou, couleur interne de la pulpe, couleur de la peau, facilité d'épluchage» ont indiqué des grands groupes de proximité. Ces résultats appuient ceux de Kumba et al. (2012) et Agre et al. (2015) qui ont souligné l'importance de ces traits dans la variation entre les accessions et dans le choix des variétés.

Les différentes variétés ont montré une résistance forte ou modérée aux maladies du manioc au même titre que la variété locale. Ainsi, les variétés biofortifiées en bêtacarotène et la variété locale sont tous sensibles aux mêmes parasites. Ces résultats sont en accord avec ceux de Lokko et al. (2006) qui avaient déjà montré que la mosaïque du manioc, la ruine bactérienne, l'acarien vert et l'anthracnose sont des parasites auxquels le manioc éprouve une certaine vulnérabilité. Ils constituent une contrainte importante dont il faut tenir compte pour optimiser la productivité des variétés à vulgariser. Toutefois, la sensibilité faible ou modérée des différentes variétés prouve qu'elles sont bien adaptées aux conditions phytosanitaires de la région Sud du Bénin. Cependant, le fait que les différentes variétés sont plus attaquées au deuxième mois par les acariens verts qu'aux premier et troisième mois peut s'expliquer par une réponse physiologique de ces variétés le deuxième mois après l'attaque par les acariens (Fagbemissi et al., 2002). La variété locale améliorée ne s'est, en aucun moment, montrée plus affectée que les 15 variétés jaunes. Il s'avère seulement nécessaire de biofortifier cette variété locale afin de la rendre plus nutritive, comme le stipulent Gannon et al. (2016) et Tanumihardjo (2011, 2013). Ainsi, la performance agronomique des variétés biofortifiées telles que I011412, I070539, I090090, I083594 et I090151 de même que la résistance de ces variétés face aux parasites du manioc font d'elles les variétés introduites les mieux adaptées aux conditions agroécologiques du Sud-Bénin. Elles sont donc des variétés intéressantes pour des expérimentations en milieu paysan dans une approche participative.

\section{Conclusion}

L'étude a permis de mettre en évidence la variabilité des caractères physicomorphologiques avec un accent particulier sur le rendement à l'hectare et la teneur en bêtacarotène des racines de manioc biofortifiées de l'IITA par rapport à une variété améliorée 
locale déjà adoptée au Bénin. Plusieurs variétés riches en bêta-carotène ont donné un rendement en racines fraîches supérieur comparativement à la variété locale améliorée RB89509. Toutes les variétés biofortifiées ont une teneur en bêta-carotène plus élevée que la RB89509. Il ressort de cette étude cinq meilleures variétés de manioc riches en bêtacarotène et à haut rendement pouvant être évaluées dans les différentes zones agroécologiques du Bénin auprès des producteurs. Ces performances devraient s'accompagner d'une évaluation technologique et physicochimique afin de sélectionner un peu plus en fonction des aptitudes à la confection des dérivés et aliments affectionnés par les populations du Bénin. De même, des études de perception permettront d'établir des critères d'acceptation et de préférence pour faciliter la diffusion de nouvelles variétés de manioc. Ainsi, la diversité des variétés de manioc en collection au Bénin serait enrichie.

\section{CONFLIT D'INTERETS}

Les auteurs déclarent n'avoir aucun conflit d'intérêts.

\section{CONTRIBUTIONS DES AUTEURS}

AKAD a supervisé l'ensemble du travail, a contribué à la collecte des données, a réalisé la saisie des données, a analysé les données et a contribué à la rédaction du manuscrit. NIO a collecté les données sur le terrain. AA a supervisé le travail et contribué à la rédaction du manuscrit.

\section{REMERCIEMENTS}

Cette étude a reçu un appui matériel, financier et technique de l'Institut International d'Agriculture Tropicale (IITA) d'Ibadan au Nigeria à travers le projet HarvestPlus. L'IITA-Ibadan a mis le matériel biologique à notre disposition pour cette étude.

\section{REFERENCES}

Adjanohoun A, Allagbe M. 2011. Référentiel Technico-économique: pour une meilleure production du manioc au sud et au centre du Bénin. INRAB/MAEP, ISBN : 978-99919- 380- 2- 8, ISSN : 1840-5479, Dépôt légal $\mathrm{n}^{\circ} 5045$ du 23/01/2011, $2^{\text {ème }}$ Trimestre 2011. Bibliothèque Nationale $(\mathrm{BN})$ du Bénin ; $46 \mathrm{p}$.
Adjanohoun A. 2006. Détermination des doses d'azote, de phosphore et de potassium pour l'accroissement des rendements et la rentabilité du manioc au Sud-Bénin. Bulletin de la Recherche Agronomique du Bénin, 51: 37-45.

Agbodjato NA, Noumavo PA, Adjanohoun A, Dagbenonbakin G, Atta M, Falcon Rodriguez A, de la Noval Pons BM, Baba-Moussa L. 2015. Response of maize (Zea mays L.) crop to biofertilization with plant growth promoting rhizobacteria and chitosan under field conditions. Journal of Experimental Biology and Agricultural Sciences., http://dx.doi.org/10.18006/2015.3 (6).566-574.

Agre AP, Dansi A, Rabbi IY, Battachargee R, Dansi M, Melaku G, Augusto B, Sanni A, Akouegninou A, Akpagana K. 2015. Agromorphological Characterization of elite cassava (Manihot esculenta Crantz) cultivars collected in Benin. Int. J. Curr. Res. Biosci. Plant Biol., DOI: http://doi.org/10.15580/GJAS.2016.8.06 2716107.

Ahanhanzo C. 2009. Contribution à l'optimisation des conditions de culture in vitro de deux espèces d'Ocimumspp. (Lamiaceae) et étude de l'influence des manipulations in vitro sur la teneur et la qualité de leur acide désoxyribonucléique (ADN). Rev. Cames-Série A., 9. http://www.sciencedirect.com.science/ar ticle/SO308814611013628. 2009.9.

Aïhou K. 2003. Interaction between organic input by Cajanus cajan and inorganic fertilization to maize in the derived savanna of the Benin Republic. Thèse de Doctorat. Universtité d'Abomey-Calavi. $114 \mathrm{p}$.

Amoussa-Hounkpatin W, Mouquet-Rivier C, Dossa R, Picq C, Avallone S. 2012. Contribution of plant-based sauces to the vitamin A intake of young children in Benin. Food Chemistry, 131: 948955.

https://doi.org/10.1016/j.foodchem.2011 .09 .085 .

Araújo DD, Amorim AB, Saleh MAD, Curcelli F, Perdigon PL, Bicudo SJ, Berto DA. 2016. Nutritional evaluation of integral cassava root silages for 
growing pigs. Animal Nutrition, 2:149153. https://doi.org/aninu.2016.04.006;

Bakayoko S, Kouadio KKH, Soro D, Tschannen A, Nindjin C, Dao D, Girardin O. 2012. Rendements en racines frais et teneurs en matière sèche de soixante-dix nouvelles variétés de manioc (Manihot esculenta Crantz) cultivées dans le centre de la Côte d'Ivoire. Journal of Animal \& Plant Sciences., 14(2): 1961-1977. http://www.m.elewa.org/JAPS; ISSN $2071-7024$.

Cacai GHT, Ahanhanzo C, Dangou JS. 2012. Effets de différentes combinaisons hormonales sur l'organogenèse in vitro de quelques cultivars locaux et variétés améliorés de Manihot esculenta cultivées au Bénin. International Journal of Biological and Chemical Sciences. $\quad 6(4)$ : $\quad 1593-1607$. http://dx.doi/10.4314/ijbcs.v6i4.19

Ceballos H, Parkes E. 2015. Measuring Provitamin A Content in Crops The 2nd Global Conference on Biofortification: Getting Nutritious Foods to People. conference brief. \#6a.

Dansi A, Adjatin A, Adoukonou-Sagbadja H, Adomou A, Falade V, Yedomonhan H, Akpagana K, De Foucault B. 2008. Traditional leafy vegetables in Benin: Folk nomenclature, species under threat and domestication Dans: Biodiversité des légumes-feuilles traditionnels consommés au Bénin. Bibliothèque nationale, Bénin. p 173.

Djoulde DR. 2005. Mise au point d'un ferment mixte destiné à la bioconversion des racines de manioc cyanogène. Thèse de doctorat, Ngaoundere. http://tel.archivesouvertes.fr/docs/00/04/ 82/82/PDF/tel-00009811.pdf.

Fagbemissi RC, Coulibaly O, Hanna R, Endamana D. 2002. Adoption de variétés de manioc et efficacité durable de la lutte biologique contre l'acarien vert du manioc au Bénin. Bulletin de la Recherche Agronomique du Bénin. Numéro 38 -Décembre 2002.

Gannon BM, Pungarcher I, Mourao L, Davis CR, Simon P, Pixley KV, Tanumihardjo SA. 2016. 13C Natural abundance of serum retinol is a novel biomarker for evaluating provitamin A carotenoidbiofortified maize consumption in male mongolian gerbils. J. Nutr., 146(7): 1290-1297.

DOI: 10.3945/jn.116.230300. Epub. Jyn 8.

Hefferon KL. 2015. Nutritionally Enhanced Food Crops; Progress and Perspectives. International Journal Molecular Sciences, 16 : 3895-3914 ; DOI: 10.3390/ ijms 16023895 .

Kouadio KKH, Ettien DJB, Bakayoko S, Soro D, Girardin O. 2014. Variabilité physico-morphologique des racines tubéreuses de manioc cultivées sur ferralsol en zone de foret d'Afrique de l'Ouest. Journal of Applied Biosciences, $82: 7316$ - 7325. ISSN 1997-5902. http://dx.doi/10.4314/ab.82i1.1

Kumba K. 2012. Genetic characterization of exotic and landraces of cassava in Ghana. Master of Sciences thesis, in Agronomy, Kwamen krumah University of Science and Technology. 111p.

La Frano MR, Woodhouse LR, Burnett DJ, Burri BJ. 2013. Biofortified cassava increases $\beta$-carotene and vitamin $A$ concentrations in the TAG-rich plasma layer of American women. Br. J. Nutr., 110, 310-320. http://doi.org/10.1017/ S0007114512005004

Songre-Ouattara LT, Gorga K, Bationo F, Savadogo A, Diawara B. 2016. Utilisation du moringa, de la spiruline, de la patate douce à chair orange et d'un complexe minéral et vitaminique dans la fabrication de biscuits de sorgho enrichis destinés aux jeunes enfants. International Journal of Biological and Chemical Sciences, 10(4): 1651-1665. http://dx.doi.org/10.4314/ijbcs.v10i4.17.

Leyva-Guerrero E, Narayanan NN, Ihemere U, Sayre RT. 2012. Iron and protein biofortification of cassava: Lessons learned. Curr. Opin. Biotechnol., 23: 257-264.

Lokko Y, Dixon A, Offei S, Danquah E, Fregene M. 2006. Assessment of genetic diversity among African cassava Manihot esculenta Grantz accessions resistant to the cassava mosaic virus disease using SSR markers. Genetic Resources and Crop Evolution, 53: 1441-1453. Doi: 10.1007/s10722-0056841-x.

Michaud DS, Feskanich D, Rimm EB. 2000. Intake of specific carotenoïds and risk of lung cancer in 2 prospective US cohorts. 
Am J Clin Nutr., 72(4): 990-997. Doi : 10.109/ajen/72.4.990.

Mokemiabeka S, Dhellot J, Kobawila SC, Louembe D. 2011. Traditional retting of cassava roots in the ponds or the rivers. International Journal of Biological and Chemical Sciences, 5(1): 134-141. http://dx.doi.org/10.4314/ijbcs.v5i1.680 91.

N'zué B, Zohouri PG, Sangaré A. 2004. Performances agronomiques de quelques variétés de manioc (Manihot esculenta Crantz) dans trois zones agroclimatiques de la Côte d'Ivoire. Agronomie africaine XVI : 1-7. http://dx.doi.org/10.4314.aga.v16i2:164 6

Olugbemi TS, Mutayoba SK, Lekule FP. 2010. Effect of Moringa (Moringa oleifera) inclusion in cassava based diets fed to broiler chickens. Int. J. Poult. Sci., 9(4): 363-367. Doi 10.5713/agas.15.0642.

R Development Core Team. 2016. R: a language and environment for statistical computing. R Foundation for Statistical Computing, Vienna, Austria.www.rproject.org.

Sahouegnon HH, Mitchikpe EC, Kayode PP, Dossa RAM. 2014. Contribution du manioc à l'alimentation et à la nutrition des enfants dans la commune de Djidja au Bénin. International Journal of Biological and Chemical Sciences, 8(4): 1757-1770. http://dx.doi.org/10.4314/ijbcs.v8i4.34

Salvador EM, Steenkamp V, McCrindle CME. 2014. Production, consumption and nutritional value of cassava (Manihot esculenta, Crantz) in Mozambique: an overview. Journal of
Agricultural Biotechnology and Sustainable Development, 6(3): 29-38. http://doi.org/10.5897/JABSD2014.0224 Sayre R, Beeching JR, Cahoon EB, Egesi C, Fauquet C, Fellman J, Fregene M, Gruissem W, Mallowa S, Manary M. 2011. The BioCassava plus program: Biofortification of cassava for subSaharan Africa. Annu. Rev. Plant Biol., 62: 251-272. doi.10.1146/annurevarplant-042110-103751.

Silva MAA, Furlan AC, Moreira I, Paiano D, Scherer C, Martins EN. 2008. Nutritional evaluation and performance of cassava root silage with or without whole soybean in for nursery pigltes. Rev Bras Zootec., 37(8): 1441-1449. http://dx.doi.org/10.1590/S151635982008000800015.

Tanumihardjo SA, Palacios N, Pixley KV. 2010. Provitamin A carotenoidbioavailability : what really matters ? 80(4-5): $336-350$. DOI: $10.1024 / 0300-$ 9831/a000042.

Tanumihardjo SA. 2011. Vitamin A: biomarkers of nutrition for development. Am. J. Clin. Nutr. Res., 94(2): 658-665. Doi: 10.3945/ajcn.110.005777.

Tanumihardjo SA. 2013. Vitamin A and bone health: the balancing act. J. Clin. Densitom., $\quad$ 16(4): 414419.doi:10.1016/j.jocd.2013.08.016

Wydra K, Verdier V. 2002. Occurence of cassava diseases in relation to environmental, agronomic and plant characteristics. Agriculture, Ecosystems \& Environment, 93: 211-226. http://doi.org/10.1016/S0167-8809 (01)00349-8. 